COMMUNICATION

\title{
Polymer supported enantioselective reactions. III. Protonation of lithioenamine derivatives of racemic 2-methylcyclohexanone
}

\author{
Colin R. McArthur,' Ji-Long Jiang, and Clifford C. Leznoff ${ }^{1}$ \\ Department of Chemistry, York University, Toronto, Ont., Canada M3J IP3
}

\begin{abstract}
Colin R. McArthur, Ji-Long Jiang, and Clifford C. Leznoff. Can. J. Chem. 60, 2984 (1982).
Asymmetric protonations, with enantioselectivities ranging to $90 \%$, were observed in reactions of lithioenamines derived from imines of racemic 2-methylcyclohexanone and insoluble cross-linked copolymers of polystyrene and divinylbenzene substituted with $(S)$-2-aminoalkoxymethyl groups.
\end{abstract}

Colin R. Mcarthur, Ji-Long Jiang et Clifford C. Leznoff. Can. J. Chem. 60, 2984 (1982).

On a observé des protonations asymétriques, avec des sélectivités énantiométriques allant jusqu'à $90 \%$, lors de réactions de lithioénamines provenant des imines de la méthyl-2 cyclohexanone racémique avec un copolymère réticulé et insoluble de polystyrène et de divinylbenzène portant des groupes aminoalkoxyméthyle-2(S).

[Traduit par le journal]

Recently we reported the use of insoluble crosslinked copolymers of styrene and divinylbenzene with covalently bonded $(S)$-2-aminoalkoxy groups (1) in the asymmetric methylation of the lithioenamines derived from cyclohexanone and 1 . The polymeric system afforded higher enantioselectivities than were observed through the use of analogous systems, under comparable conditions, in solution ( 1 ).

We now report preliminary results in our study of asymmetric protonations of the lithioenamines derived from the polymer-bound ketimines 2 formed from reaction of 1 with racemic 2-methylcyclohexanone (pathway A).

Two examples of nonbiological systems involving asymmetric protonations, conducted in the solution phase, have been reported recently. Thus, amino-acids may be "deracemized" to $70 \%$ e.e. (2), and a bicyclobutane bridged enolate anion has been enantioselectively protonated to $48 \%$ e.e. (3).

In our studies, to distinguish between asymmetric protonation of the lithioenamines from 2 and a bias in the reaction of enantiomers during imine formation from racemic 2-methylcyclohexanone, imines $\mathbf{2}$ were subjected to direct hydrolysis (pathway $B$ ). The degree of resolution depends on the structure of 1 . Thus, 2-aminopropoxymethylated polystyrene (1a) led to no apparent resolution, while the 3-phenyl derivative $(1 b)$ afforded the 2-methylcyclohexanones with $50 \%$ excess of the $(S)$-enantiomer (Table 1). While $2 b$, then, must have $(S)$-2-methylcyclohexylidene moieties in $50 \%$

'Authors to whom correspondence may be addressed.

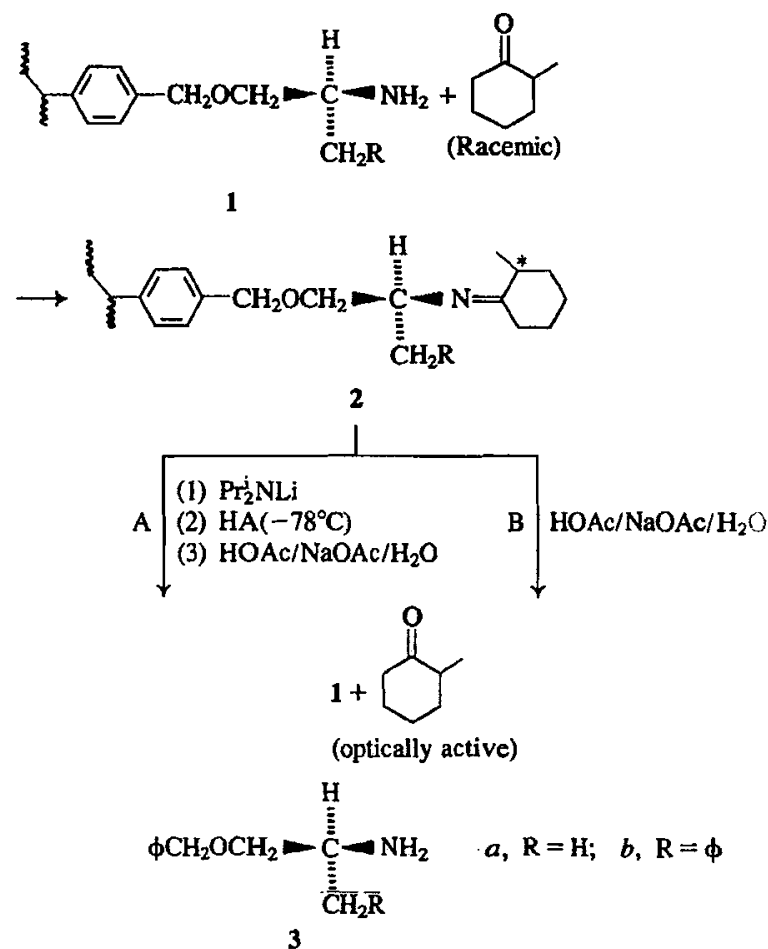

e.e., this chiral centre would be expected to be lost on deprotonation at the 2-position if a planar lithioenamine is assumed (see below).

The enantioselectivity of pathway A was found to be highly dependent on both the structure of the asymmetric inducing moiety on the polymer, and on the structure of the protonating species (Table 1). Bias is moderately towards formation of excess 
TABLE 1. Enantiomeric excesses of product 2-methylcyclohexanone

\begin{tabular}{|c|c|c|c|}
\hline & & \multicolumn{2}{|c|}{$\begin{array}{l}\text { Enantiomeric excess } \\
\quad(R) \text { or }(S)(\%)^{b}\end{array}$} \\
\hline & & $\mathrm{R}=\mathrm{H}$ & $\mathrm{R}=\phi$ \\
\hline HA in Pathway A & $\begin{array}{l}\mathrm{HOAc} \\
\mathrm{Et}_{3} \mathrm{NHCl} \text { in THF } \\
2,6-\mathrm{Di}-t \text {-butyl } p \text {-cresol in THF } \\
t \text {-BuOH } \\
\mathrm{EtOH}+\mathrm{Et}_{3} \mathrm{NHCl} \text { in THF } \\
\text { EtOH }\end{array}$ & $\begin{array}{c}20(R) \\
36(R) \\
8(R) \\
- \\
\overline{-} \\
22(R)\end{array}$ & $\begin{array}{c}22(S) \\
\overline{-} \\
\overline{0} \\
82(S) \\
90(S)\end{array}$ \\
\hline Pathway B & & 0 & $50(S)$ \\
\hline
\end{tabular}

(R)-2-methylcyclohexanone using $1 a$, but more strongly towards $(S)$-2-methylcyclohexanone using $1 b$ (of the same chirality). It is noteworthy that, in the case of $1 b$, bond formation to the proton must have occurred on the side of the 2-position opposite to that in bond formation to carbon in the methylation reactions described earlier (1) in order for both to lead to $(S)$-2-methylcyclohexanone.

Most significant are two extremum results. $(S)$-2Methylcyclohexanone was obtained in $90 \%$ e.e. when the lithioenamine from $2 b$ was protonated using ethanol, but only racemic product was obtained using tert-butyl alcohol, after the resulting polymers were hydrolyzed under the same conditions. These observations are consistent with regioselective deprotonation of $2 b$ at the 2-position to form a planar lithioenamine followed by highly diastereoselective $C$-protonation when ethanol is used, or nondiastereoselective selective protonation with tert-butyl alcohol. Predominant deprotonation at the more highly alkylated $\alpha$-position has been observed in other ketimines $(4,5)$ including those of cyclohexanone imines (4) using alkylithium reagents. In view of the studies of Knorr and Löw (6) the $C$-protonation may be a result of a tautomerization ${ }^{2}$ following an initial kinetically controlled $N$-protonation to form an enamine.

Another possibility may be deprotonation at the 6-position of the 2-methylcyclohexanone imine system, as observed by Meyers et al. (7) in reactions of lithium diisopropylamide on imines in solution, followed by a complex equilibration prior to hydrolysis. ${ }^{2}$

In any event, diastereoselective $C$-protonation at the 2-position ultimately must have occurred prior to hydrolysis by a mechanism that is highly influenced by the structure of the protonating species.

An analogous system in solution was investigated. The imine from racemic 2-methylcyclohexanone and $(S)$-2-aminopropyl benzyl ether ( $3 a$ )

${ }^{2}$ We appreciate these alternative explanations suggested by one referee.
(1) was treated under the same conditions as those in pathway $A$ for the polymeric system. The product exhibited no optical activity. We plan to examine the analogous system derived from $(S)-2-$ amino-3-phenylpropyl benzyl ether (3b).

Our result of $90 \%$ e.e. would appear to be unprecedented in enantioselective protonation reactions in nonbiological systems. These results are consistent with earlier observations (1) of enhancement of enantioselectivities through the use of polymer supported asymmetric inducing agents relative to analogous systems in solution. While mechanisms have been proposed to explain the predominant enantioselectivities in related reactions $(9,10)$ additional studies are required to rationalize the results that we report here. Studies in this area are continuing.

\section{Acknowledgements}

We thank the Natural Sciences and Engineering Research Council of Canada and the Faculty of Science, York University for financial support of this research.

1. (a) C. R. MCARTHUR, P. M. WORSTER, J.-L. JiANG, and C. C. Leznoff. Can. J. Chem. 60, 1836 (1982); (b) P. M. Worster, C. R. MCARThUR, and C. C. LeZNOFF. Angew. Chem. Int. Ed. Engl. 18, 221 (1979).

2. L. Duhamel and J. C. Plaquevent. Tetrahedron Lett. 21, 2521 (1980).

3. H. Hogeveen and L. ZwarT. Tetrahedron Lett. 23, 105 (1982).

4. A. Hosomi, Y. Arakı, and H. Sakurai. J. Am. Chem. Soc. 104, 2081 (1982).

5. J. K. SMith, D. E. Bergbreiter, and M. NewComb. J. Org. Chem. 46, 3157 (1981).

6. R. KNORR and P. Löw. J. Am. Chem. Soc. 102, 3241 (1980).

7. A. I. Meyers, D. R. Williams, G. W. ERICKSON, S. White, and M. Druelinger. J. Am. Chem. Soc. 103, 3081 (1981).

8. J. K. Whitesell and M. A. Whitesell. J. Org. Chem. 42, 377 (1977).

9. A. I. Meyers, D. R. Williams, and M. Druelinger. J. Am. Chem. Soc. 98, 3032 (1976); A. I. MEYERS and D. R. Williams. J. Org. Chem. 43, 3245 (1978).

10. R. R. Fraser, F. AKIYAMA, and J. BanviLle. Tetrahedron Lett. 3929 (1979). 